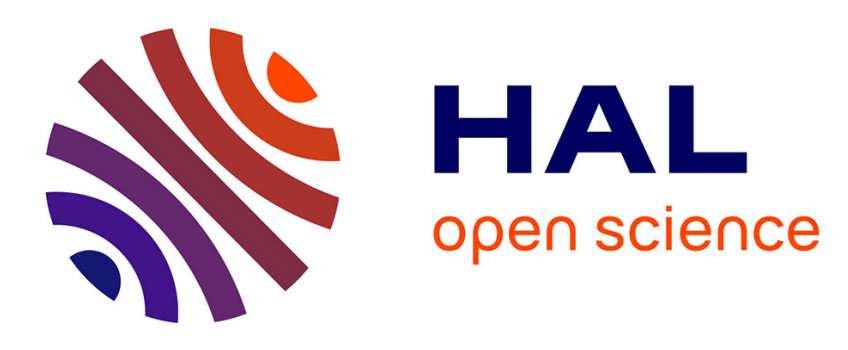

\title{
Region Growing Structuring Elements and New Operators Based on Their Shape
}

\author{
Vincent Morard, Etienne Decencière, Petr Dokládal
}

\section{To cite this version:}

Vincent Morard, Etienne Decencière, Petr Dokládal. Region Growing Structuring Elements and New Operators Based on Their Shape. Signal and Image Processing (SIP 2011), Dec 2011, Dallas, United States. pp.Pattern recognition, track 759-018. hal-00834502

\section{HAL Id: hal-00834502 \\ https:/ /hal-mines-paristech.archives-ouvertes.fr/hal-00834502}

Submitted on 15 Jun 2013

HAL is a multi-disciplinary open access archive for the deposit and dissemination of scientific research documents, whether they are published or not. The documents may come from teaching and research institutions in France or abroad, or from public or private research centers.
L'archive ouverte pluridisciplinaire HAL, est destinée au dépôt et à la diffusion de documents scientifiques de niveau recherche, publiés ou non, émanant des établissements d'enseignement et de recherche français ou étrangers, des laboratoires publics ou privés. 


\title{
REGION GROWING STRUCTURING ELEMENTS AND NEW OPERATORS BASED ON THEIR SHAPE
}

\author{
Vincent Morard and Etienne Decencière and Petr Dokládal \\ Centre de Morphologie Mathématique \\ Mathématiques et Systèmes, MINES ParisTech; \\ 35, rue Saint-Honoré, 77305 Fontainebleau CEDEX - France \\ \{Vincent.Morard;Etienne.Decenciere;Petr.Dokladal\}@ mines-paristech.fr
}

\begin{abstract}
This paper proposes new adaptive structuring elements in the framework of mathematical morphology. These structuring elements (SEs) have a fixed size but they adapt their shape to the image content by choosing, recursively, similar pixels in gray-scale, with regard to the seed pixel. These new SEs are called region growing structuring elements (REGSEs).

Then, we introduce an original method to obtain some features by analyzing the shape of each REGSE. We get a powerful set of operators, which is able to enhance efficiently thin structures in an image.

We illustrate the performance of the proposed filters with an application: the detection of cracks in the framework of non-destructive testing. We compare these methods with others, including morphological amoebas and general adaptive neighborhood structuring elements and we see that these operators, based on REGSE, yield the best detection for our application.
\end{abstract}

\section{KEY WORDS}

Image Enhancement, Pattern Recognition, Mathematical Morphology, Region Growing Structuring Elements, Crack Detection, Inertia Operator.

\section{Introduction}

In the framework of mathematical morphology [11], feature enhancement and noise reduction are two tasks of great importance. This work is a part of an industrial project where our goal is to extract automatically all crack-like defects from metallic pieces. These cracks are long, narrow and the approach used in this paper, filters out the noise while preserving the entire crack. Classical translation invariant morphology uses SEs of a fixed shape, to probe an image at different places. This rigid shape turns out to be a disadvantage for enhancing curvilinear thin structures. Therefore, adaptive structuring elements constitute an elegant solution to our problem. These SEs are called structuring functions by Serra in [12], where he defined the erosion and the dilation with adaptive structuring elements.

In the literature, there are several ways to suppress the translation invariance of classical SEs. The first one consists in adjusting their sizes and shapes to their location in the image as used by Beucher et al. in [1], in a traffic camera control application. The size of the SEs depends on the perspective and varies linearly with the vertical position of the vehicle in the image.

Another possibility is to adapt the morphology of the SEs to the image content. Gordon and Rangayyan, in [7], introduced the notion of Adaptive Neighborhood. The size of the SEs is chosen by maximizing a contrast function for each pixel. This led Braga-Neto, in [4] and later, Debayle and Pinoli, in [6], to present the General Adaptive Neighborhood Structuring Elements (GANSE). The structuring elements are defined in terms of $m^{\text {th }}$ order connectivity. Another approach was followed by Lerallut et al. in [9]. They proposed the morphological amoebas, which are balls defined with a special geodesic distance. Recently, Grazzini and Soille took the same principle and introduced structuring elements using the notion of geodesic time [8]. In [13], Tankyevych et al. worked with linear SEs to detect thin structures, which adapt their orientations to the local orientation of the objects. Alternative approaches were introduced by Salembier in [10], Cheng and Venetsanopoulos in [5] and others. The theoretical aspects of the adaptive structuring elements were studied by Bouaynaya et al. in $[2,3]$.

Among the different approaches proposed in the literature, morphological amoebas introduced by Lerrallut et al. appeared as a promising solution. The amoebas tend to have a circular shape but can adapt locally to the image content. Their shapes are driven by a generalized, gradient-weighted, distance. Let $\sigma=$ $\left\{x_{0}, \ldots, x_{i}, x_{i+1}, \ldots, x_{n}\right\}$ be a path between two points $x_{0}$ and $x_{n}$. The length of this path is defined by $L(\sigma)=$ $\sum_{1 \leq i \leq n}\left(1+\lambda d_{\text {pixel }}\left(x_{i-1}, x_{i}\right)\right)$ where $d_{\text {pixel }}$ is the grayscale difference between two pixels and $\lambda$ the gradient penalty parameter. Therefore, the amoebas distance between two pixels $x$ and $y$ is defined as follows:

$$
d_{\lambda}(x, y)=\min _{\sigma \in P(x, y)} L(\sigma),
$$

where $P(x, y)$ is the set of paths linking $x$ and $y$. In our framework, it is necessary to continue further in the same idea, to relax any shape constraints for the sake of total adaptability to the image content. Debayle and Pinoli have followed the same idea, where the SEs have no shape and 
no size constraints. The only parameter $m \in \mathcal{Z}^{+}$controls the homogeneity of the SE. Let $x$ be the central pixel and $x_{i}$ be a pixel connected to $x$ with a path $\sigma$, such that $\forall x_{i} \in \sigma,\left|f\left(x_{i}\right)-f(x)\right| \leq m$. Hence, the SE is a connected component composed of pixels $x_{i}$ connected by a path to $x$. The complexity of this algorithm depends on the image content and is potentially very high as the SE can be arbitrary large. Moreover, this filter does not remove impulsive noise since strong gradients prevent the growth of the SEs.

Therefore, we impose a size constraint to address these drawbacks and to obtain interesting operators capable of filtering or feature enhancement. These new SEs are called region growing structuring elements (REGSE).

With REGSEs, the SEs have no shape constraint, which is fully driven by the image content. Hence, by analyzing the shape of each REGSE, we get some information on the underlying structures. To the authors' knowledge, this is the first time that the shape of the adaptive structuring elements is used to assess features.

Section 2 introduces the concept, the definition and the properties of REGSEs. Section 3 presents the definition of a new set of operators, which is based on the shape analysis of the adaptive SEs. Then, section 4 focuses on applications that highlight both the noise reduction and the feature enhancement skills of these operators. Finally, section 5 is reserved to the practical considerations of this method, where we propose an implementation example.

\section{REGSE : adaptive SE}

\subsection{Definition}

Let $f$ be a gray-scale image, $f: D \rightarrow V$ with $D \subset \mathcal{Z}^{2}$, the domain and $V=[0, \ldots, M]$, the set of values. For all $x \in D$ let $\mathcal{A}_{x}$ be the set of SEs such that $\mathrm{A}_{x} \in \mathcal{A}_{x}$ if and only if:

$$
\begin{aligned}
& \mathrm{A}_{x} \subseteq D, \\
& x \in \mathrm{A}_{x}, \\
& \mathrm{~A}_{x} \text { connected. }
\end{aligned}
$$

Further, we shall denote by $\mathrm{A}_{x}^{N}$, a SE belonging to $\mathcal{A}_{x}$, having exactly $N$ pixels: $\operatorname{Card}\left(\mathrm{A}_{x}^{N}\right)=N$. The construction of these structuring elements will be done recursively with a region growing process: $x$ is considered as the seed and $\operatorname{Card}\left(\mathrm{A}_{x}^{N}\right)=N$ is the stopping criterion:

$$
\begin{aligned}
& \mathrm{A}_{x}^{1}=\{x\} \\
& \mathrm{A}_{x}^{2}=\mathrm{A}_{x}^{1} \cup\left\{y_{2}\right\} \\
& \vdots \\
& \mathrm{A}_{x}^{N}=\mathrm{A}_{x}^{N-1} \cup\left\{y_{N}\right\},
\end{aligned}
$$

where $y_{i}$ is one of the neighbor pixels of $\mathrm{A}_{x}^{i-1}$, selected through the minimization of a homogeneity function $\rho$, written :

$$
\forall a, b \in D, \rho(a, b)=|f(a)-f(b)| .
$$

Then, $y_{i}$ is given by:

$$
y_{i} \in \underset{y_{j} \in \mathcal{N}\left(\mathrm{A}_{x}^{j-1}\right)}{\operatorname{argmin}} \rho\left(x, y_{j}\right),
$$

where $\mathcal{N}($.$) denotes the neighborhood relation (detailed be-$ low). Figure 1 presents the shape of 4 REGSEs (white pixels generated from the crosses) on a given picture. We notice the SEs are very flexible. Thus, the structuring elements can be spread over the entire image (the third SE of figure 1 follows a constant-level curve of the input signal). We can see here the main advantage of this method, since the structuring elements can follow any thin structures, even one-pixel wide. Another important observation is that, on large flat zones, the shape of the SEs is a square (SE 1 on the top left). This is due to the region growing implementation. On flat zones, $\rho$ is equal to zero and equation 5 is reduced to arbitrarily choosing a pixel from the neighborhood of $\mathrm{A}_{x}^{N-1}:\left(y_{i} \in \mathcal{N}\left(A_{x}^{N-1}\right)\right)$. Classically, a region growing process is based on a queue. Associated to an 8 connectivity, this leads to a propagation following a square shape. In what follows, we will use this 8 connectivity, which is formally written: $\mathcal{N}(x)=\left\{y,\|x-y\|_{L_{\infty}}=1\right\}$. This ensures the regular shape of $\mathrm{A}_{x}^{N}$ on flat zones. Later, we will use this important property to build new operators based on the shape of the REGSEs (see section 3).

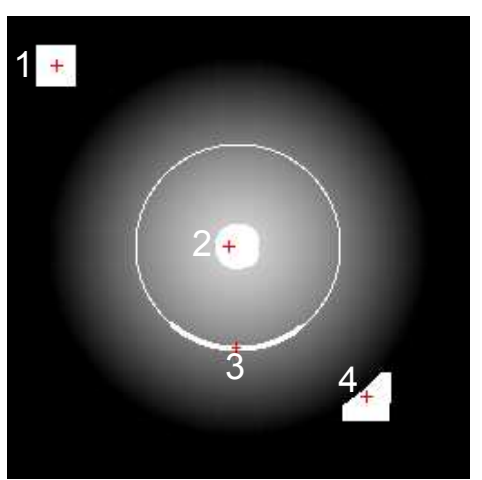

Figure 1. Four region growing structuring elements with $N=506$ pixels. The central pixels of each SE (the seeds), are referenced by the crosses.

\subsection{Mathematical morphology}

With these adaptive SEs, we are able to build the elementary operators of mathematical morphology. By taking for each pixel of the image $f$ the minimum over the REGSE, we obtain the erosion $\epsilon$ :

$$
\epsilon^{N} f(x)=\bigwedge_{x_{i} \in \mathrm{A}_{x}^{N}} f\left(x_{i}\right) .
$$

The dilation adjunct to the erosion is given by:

$$
\delta^{N} f(x)=\bigvee_{x_{i} \mid x \in \mathrm{A}_{x_{i}}^{N}} f\left(x_{i}\right) .
$$


Recall that the transposition of spatially variant SE is not a geometric set reflection (as for the translationinvariant SE), but rather the so-called set of descendants (see [12], Section 2.2 for details). Hence, using the adjunction allows to compute openings and closings, respectively written $\gamma^{N}$ and $\varphi^{N}$. We have seen that a REGSE can adapt its shape to any structure. This is a major advantage because it can follow one-pixel wide and tortuous structures. However, in some conditions, this could be considered as a drawback since thin structures could be found in the noise. Just like morphological amoebas, it is sometime useful to define a pilot image to compute the shape of the REGSE [9]. The pilot image can be the initial image blurred by a Gaussian filter of size 1 or 2. Recall that an opening (or closing) by adjunction requires the same pilot image for the erosion and for the dilation. Using a pilot image to construct REGSEs reduces the sensitivity to the noise pattern, which improves the noise reduction capabilities of this filter.

The result of an opening and a closing are presented in figures 5(b) and 5(c) with a Gaussian pilot image of standard deviation equal to 1 . These filters preserve the boundaries of the image except when the size of the REGSE is bigger than the size of the analyzed structures. Indeed, in the following section, we will see that openings and closings with REGSEs behave similarly to area openings and closings.

\subsection{Properties}

For binary images, the REGSE opening of size $N$ is equivalent to an area opening of the same size. This behavior comes from the construction of a REGSE: $\operatorname{Card}\left(\mathrm{A}_{x}^{N}\right)=$ $N$. Therefore, the REGSE must have exactly $N$ pixels and every object smaller than $N$ is suppressed. By duality, closings are equivalent to area closings.

This equivalence between a REGSE opening and an area opening is not true anymore for gray-scale images. Figure 2 points out some differences for a one-dimensional signal. Here, the area opening of size 3 does not change the input signal, whereas an opening based on REGSEs of size 3 , deletes the small step referred as pixel $x_{6}$. By construction, the REGSE of pixel $x_{6}$ is $A_{x_{6}}^{3}=\left\{x_{6}, x_{7}, x_{8}\right\}$. During the erosion process, only one pixel is changed. The pixel $x_{6}$ took the value of the pixel $x_{7}$. Afterward, the dilation by adjunction does not change anything since pixel $x_{6}$ does not belong to any others REGSEs. Here, we get $\gamma_{\text {REGSE }} \leq \gamma_{T I}$ and this is always true for 1D signals. With this example, we have noticed that this filter is useful to clean these small steps between boundaries.

At the first glance, these filters based on REGSEs seem to be connected operators. However, figure 3 gives a simple counter example where a new boundary is created during the opening process. The pixels $x_{1}, x_{2}$, and $x_{3}$ have the same adaptive structuring elements: $A_{x}^{4}=$ $\left\{x_{1}, x_{2}, x_{3}, x_{4}\right\}$ just like pixels $x_{6}, x_{7}$, and $x_{8}: A_{x}^{4}=$ $\left\{x_{5}, x_{6}, x_{7}, x_{8}\right\}$. Then, during the dilation by adjunction, the flat zone made by pixels from $x_{1}$ to $x_{3}$, propagates its value to $x_{4}$ whereas $x_{5}$ does not change. Hence, they are not connected operators.

Another property, which is not immediately intuitive, is illustrated in figure 4. An opening of size 5 is locally higher than an opening of size 6 (pixel $x_{6}$ ). This implies that the family of openings based on REGSE, is not a granulometry, as defined by Serra in [12].

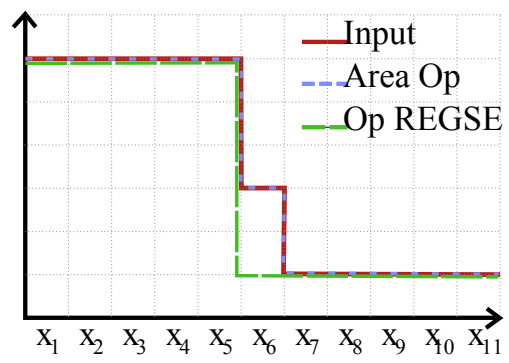

Figure 2. 1D signal and its corresponding area opening and REGSE opening. Size 3 pixels.

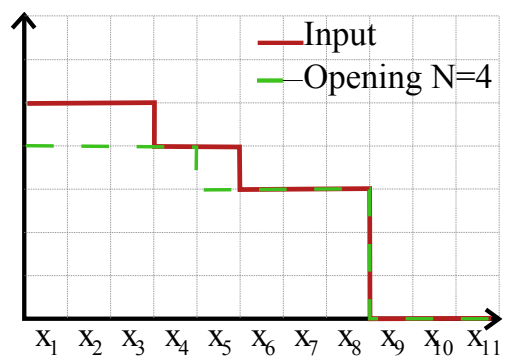

Figure 3. 1D signal and its corresponding REGSE opening of size 4 pixels.

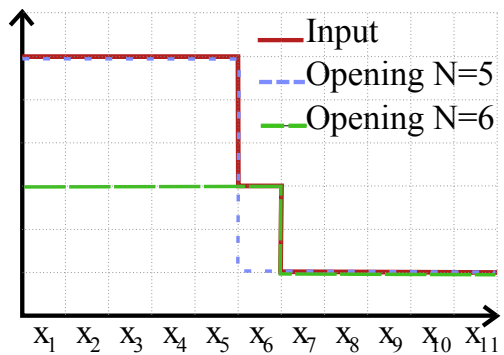

Figure 4. 1D signal and its corresponding REGSE openings of size 5 and 6 pixels.

The REGSEs have no shape constraint however, the size is fixed and the region growing process preserves a regular shape on flat zones. In the following section, new operators are introduced that analyze the shape of the adaptive SEs. 


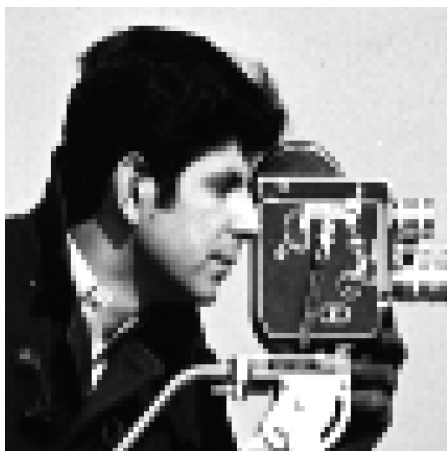

(a) Input

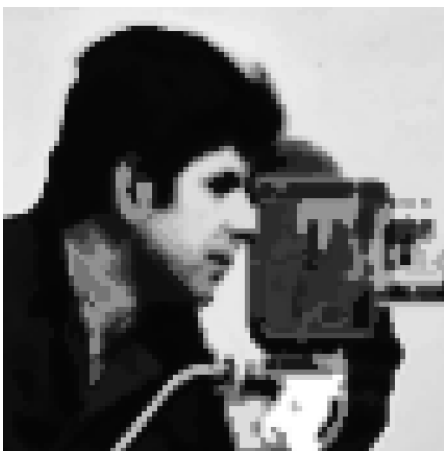

(b) Opening (REGSE of size 35)

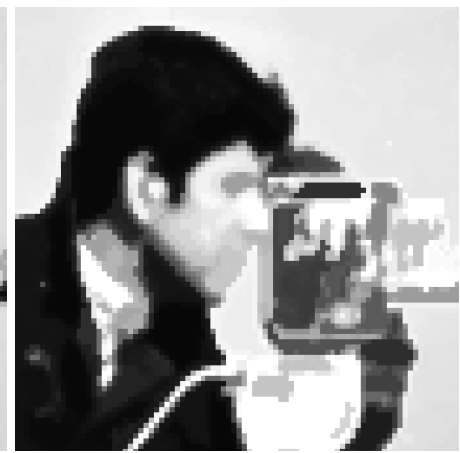

(c) Closing (REGSE of size 35)

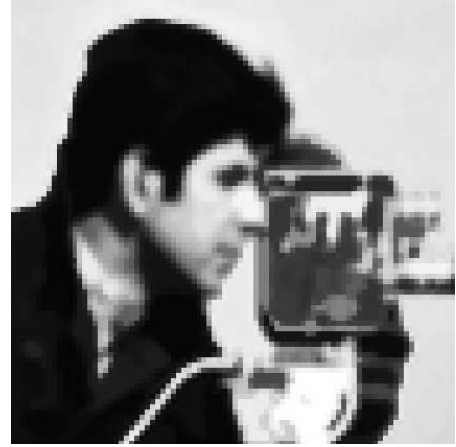

(d) Mean (REGSE of size 35)

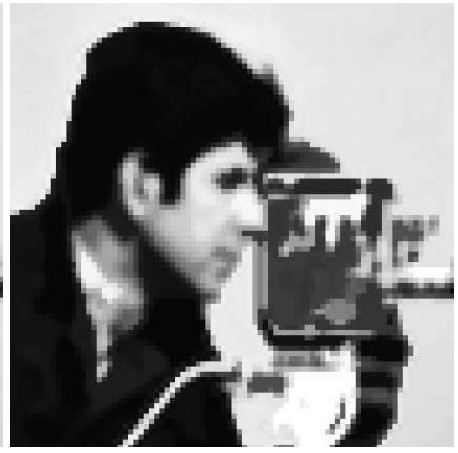

(e) Median (REGSE of size 35)

Figure 5. Noise reduction filter based on REGSEs of a size $N=35$. The pilot image is initial image blurred with a Gaussian filter of standard deviation equal to 1 .

\section{New operators based on the shape of the SEs}

A new powerful set of operators can be defined based on adaptive structuring elements. They use the shape information of each structuring element for shape-controlled feature enhancement. The proposed operators are general and can be adapted to many adaptive structuring elements. However, they are especially built for REGSEs, as their size is constrained but not their shape. Hence, we will describe these new operators using REGSEs.

\subsection{Inertia operator}

The Inertia operator computes, for each pixel, the moment of inertia of the adaptive SE:

$$
I^{N}(x)=\frac{1}{N} \sum_{x_{i} \in A_{x}^{N}}\left\|x_{i}-\bar{x}\right\|_{2}^{2},
$$

with $\bar{x}=\frac{1}{N} \sum_{x_{i} \in A_{x}^{N}} x_{i}$, the barycenter of the structuring element. Therefore, when the seed pixel is on a thin structure, the REGSE's shape will be elongated and the answer of this operator will be strong. Hence, this is a very useful operator to detect elongated structures in the image.

\subsection{Extension operator}

Another operator, namely the Extension operator, has roughly the same behavior as the inertia operator. Instead of computing the inertia of the REGSE's shape, we find the maximal distance from the REGSE's barycenter (Equation 9). This operator is dependant on the image's scale whereas the Inertia operator is scale invariant.

$$
\operatorname{Ext}^{N}(x)=\max _{\forall x_{i} \in A_{x}^{N}}\left\|x_{i}-\bar{x}\right\|_{2} .
$$

These two operators detect elongated structures in the image, no matter their gray-scale values. To focus on a given type of structures, we introduce a parameter $p$ that depends on the average gray-scale value of the structuring element. $p(x)=\frac{1}{N} \sum_{x_{i} \in A_{x}^{N}} f\left(x_{i}\right)$ to highlight bright structures and $p(x)=\frac{1}{N} \sum_{x_{i} \in A_{x}^{N}}\left(M-f\left(x_{i}\right)\right)$ for dark structures. Therefore, these weighted operators can efficiently enhance bright or dark thin structures. The usefulness of these operators is shown through examples presented in the next section.

\section{Experiments}

REGSEs can be used for many linear and non-linear operators. Here, we describe some of them for gray-scale images with a Gaussian pilot image of standard deviation of 
1. Then, we compare them with morphological amoebas and with GANSE. Finally, a real application is proposed.

\subsection{Mean, median and rank filters}

By construction, REGSEs are reluctant to cross object edges since, they are composed of pixels having similar intensity level. By replacing the output seed pixel by the mean value of $\mathrm{A}^{N}$ (mean filter), we increase the signal over noise ratio, without misplacing the boundaries of the image. The more general rank filters sort the pixel values from the REGSE, and assign the output seed pixel with a value from the sorted list of $\mathrm{A}^{N}$. The minimum value is the erosion, the maximum is the dilation and the middle value is the median filter. Little blur is introduced and like a classical median filter, the salt and pepper noise is suppressed.

Figures 5(d) and 5(e) present a mean and a median filter on the image cameraman. The noise is considerably reduced without excessively blurring the boundaries of the image.

\subsection{Comparison with other methods}

We compare the REGSE with morphological amoebas and with GANSE respectively introduced by Lerrallut et al. and by Debayle and Pinoli. These two methods are explained in the introduction, as well as the signification of the tuning parameters. Figure 6(a) is a simple material image used for illustration purposes. We want to filter out the white artifacts in the center of the cells.

With amoebas, the radius is chosen to match the number of pixels of the REGSE ( $r=5$ pixels). However, there is another parameter to work out: the gradient penalty $\lambda$ for the tonal distance. This is not an easy parameter to set. With a very small value of $\lambda$, the filter behaves just like a translation invariant SE (the image content is not taken into account). On the contrary, a high value of $\lambda$ will prevent the amoebas to grow up, unable to cross any gradient and leaving the initial image unchanged. Empirically, we choose $\lambda=0.08$. Figure 6(b) is an opening with amoebas and we notice that the noise is highly reduced. However, the boundaries are blurred and the artifacts are not removed completely.

The GANSE method involves only one parameter $m$. If $m$ is small, the GANSE is unable to grow up whereas, a high value will allow the SE to cross large gradients. However, the gradient between the artifact and the background is the same as the gradient between the cells boundaries and the background. Then, this filter is relatively inefficient to filter out the artifacts in this case.

With the REGSE, the parameterization is straightforward and the value of $N$ is chosen by analyzing the largest structures to suppress ( $N=25$ pixels). A REGSE opening, preserves correctly the boundaries, while suppressing the artifacts.

The filter based on REGSE, are able to adapt fully their shapes to the image content. Moreover, the size con-

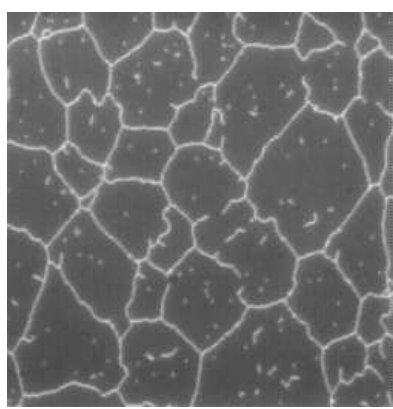

(a) Input

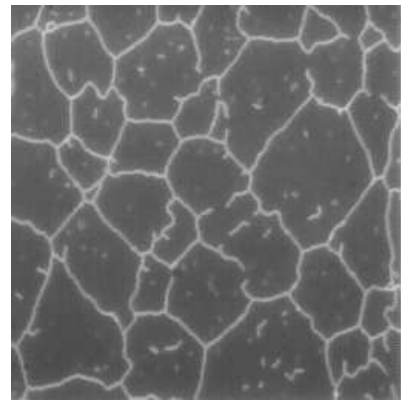

(c) GANSE $(m=35)$

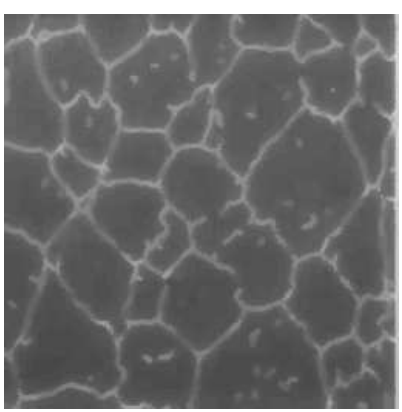

(b) Amoebas ( $r=5, \lambda=0.08)$

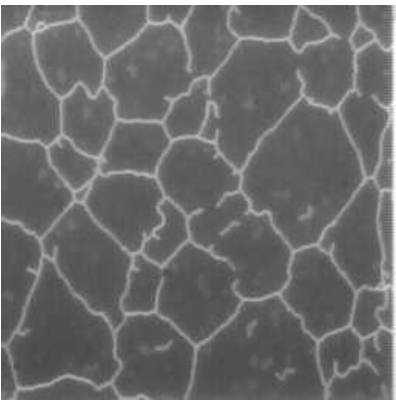

(d) $\operatorname{REGSE}(N=25$ pixels $)$
Figure 6. Comparison of openings with different adaptive structuring elements.

straint is a key parameter for feature enhancement, where this filter outperforms the other methods. In the next section, we apply these operators on a real application and we compare and discuss the performance of the operators based on REGSE.

\subsection{Application to the detection of cracks}

The idea of this filter came from picture 7(a). The extraction of these very thin cracks is easier if the structuring elements adapt their shapes to the image content. Relaxing the distance constraint adds more flexibility to the SE shape, which can now follow any crack with no restriction on its tortuosity. Therefore, an opening with REGSEs of approximately the size of the structure to detect, allows the enhancement of this structure. Figure 7 presents the results of an opening with a REGSE, with a segment of line as $\mathrm{SE}$, and with amoebas. The supremum of openings with a segment of line in all orientations allows removing the noise. However, it only enhances the straight parts of the cracks. The amoebas SEs are not able to extract thin lines whereas, an opening based on REGSE with a size of 150 pixels yields a good enhancement of these structures.

Even more interestingly, the Inertia and the Extension operators, built with the shape of each REGSE, offer a very good detection (7(e) and 7(f)). A direct threshold on the resulting image is even possible and leads to a correct segmentation and a good crack extraction. 


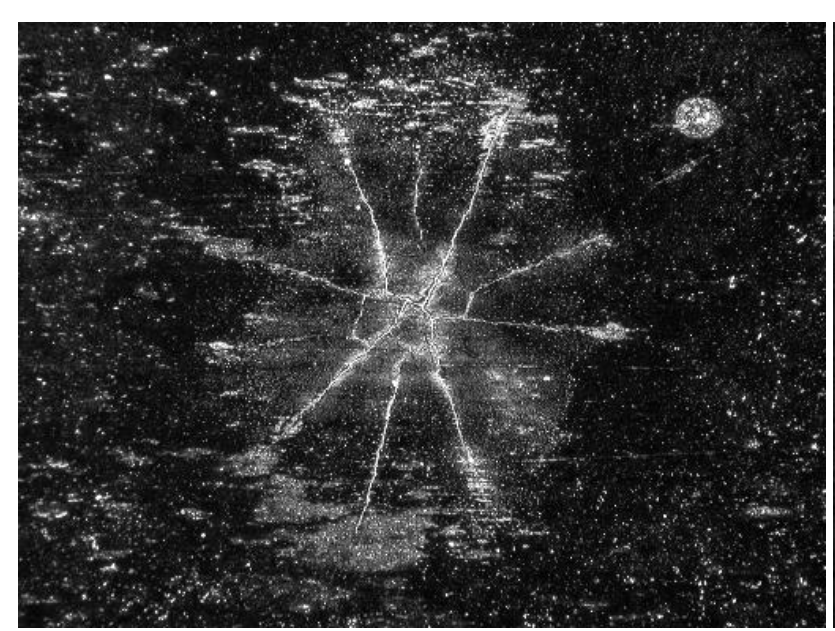

(a) Input

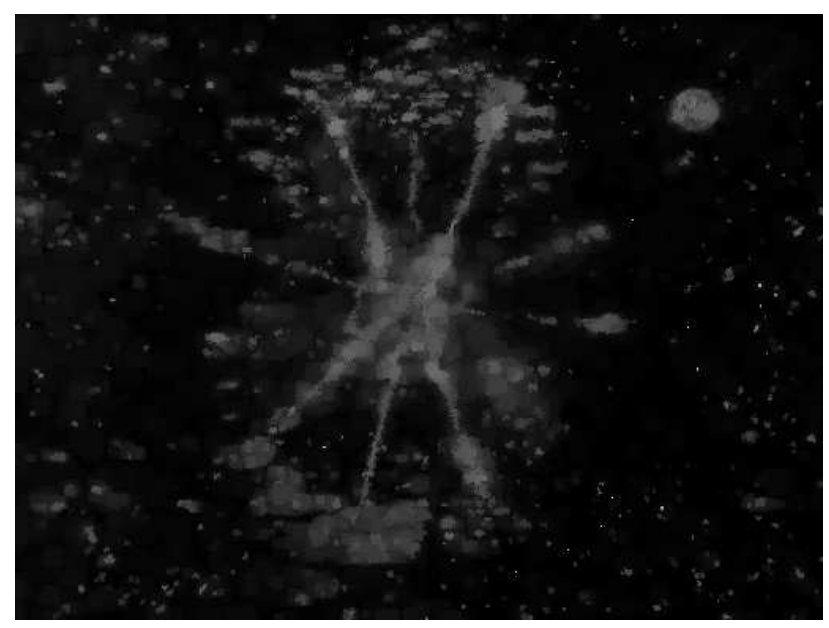

(c) Amoebas (radius of 5, $\lambda=0.025$ )

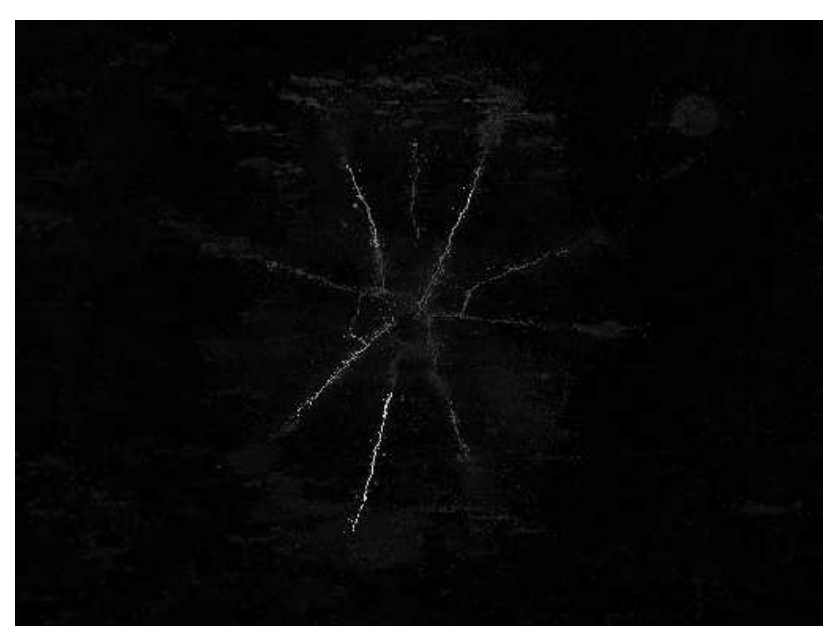

(e) Weighted inertia operator based on REGSEs, $\mathrm{N}=80$ pixels pixels

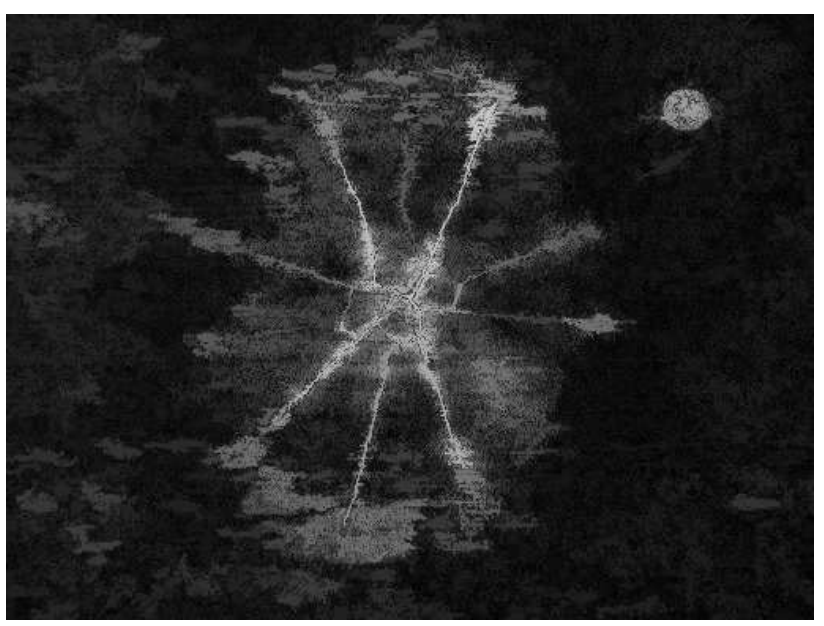

(b) Opening based on REGSEs, $\mathrm{N}=150$ pixels

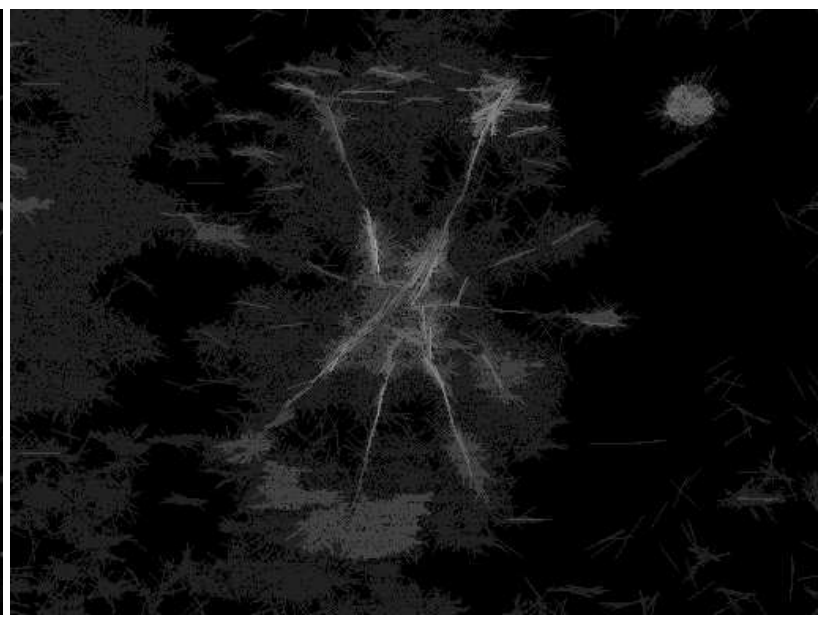

(d) Opening with rotating segments of length 21 pixels. Lines are oriented every $1^{\circ}$.

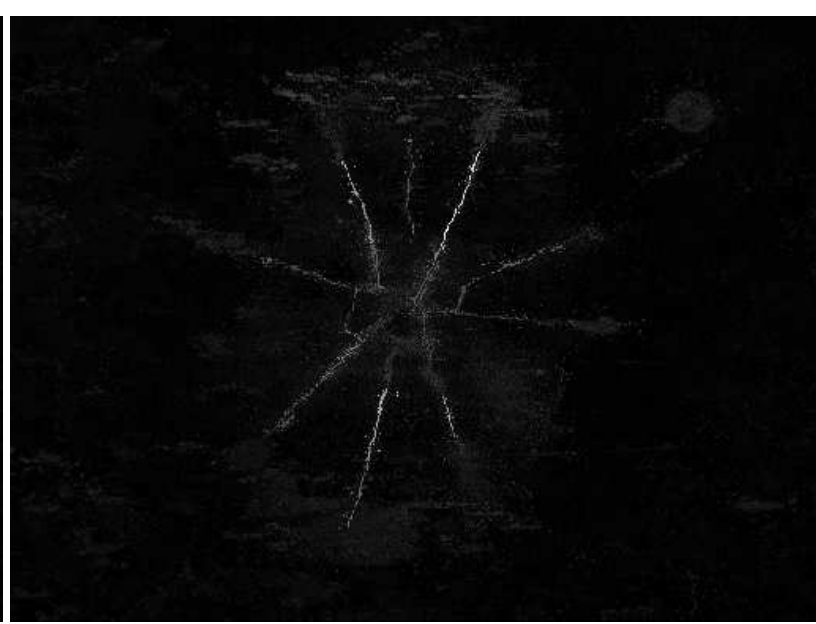

(f) Weighted maxima extension operator based on REGSEs, $\mathrm{N}=80$ pixels pixels

Figure 7. Comparison of methods with different structuring elements. 


\section{Practical considerations}

The construction of the REGSE turns out to be a computationally intensive method. Therefore, having an efficient implementation is of great importance. Equation 3 describes a recursive construction of the SE. Hence, we introduce an adapted data structure to simulate the recursion. In this section, we will speak about this data structure, and how we can apply it to create REGSEs. We will finally make some remarks on the execution time between REGSE, amoebas, and GANSE.

\subsection{Data structure}

The data structure used is a hierarchical queue (HQ). This HQ will be composed of $M$ queues (fifo) ordered by decreasing priority (One for each gray tone of $f$ ). Hence, $H Q(0)$ and $H Q(M)$ have respectively the highest and the lowest priority. The insertion of a pixel $x_{i}$ into the HQ is done with the priority $\rho\left(x, x_{i}\right)$, with $x$ being the seed pixel. Thus, the operation inserting the pixel $x_{i}$ into the $H Q$ is written $H Q\left(\rho\left(x, x_{i}\right)\right)$.push_back $\left(x_{i}\right)$ and then, removing a pixel having the highest priority is made with a pop as described in the algorithm 1 . This data structure used here is computationally efficient, since we avoid the sorting step.
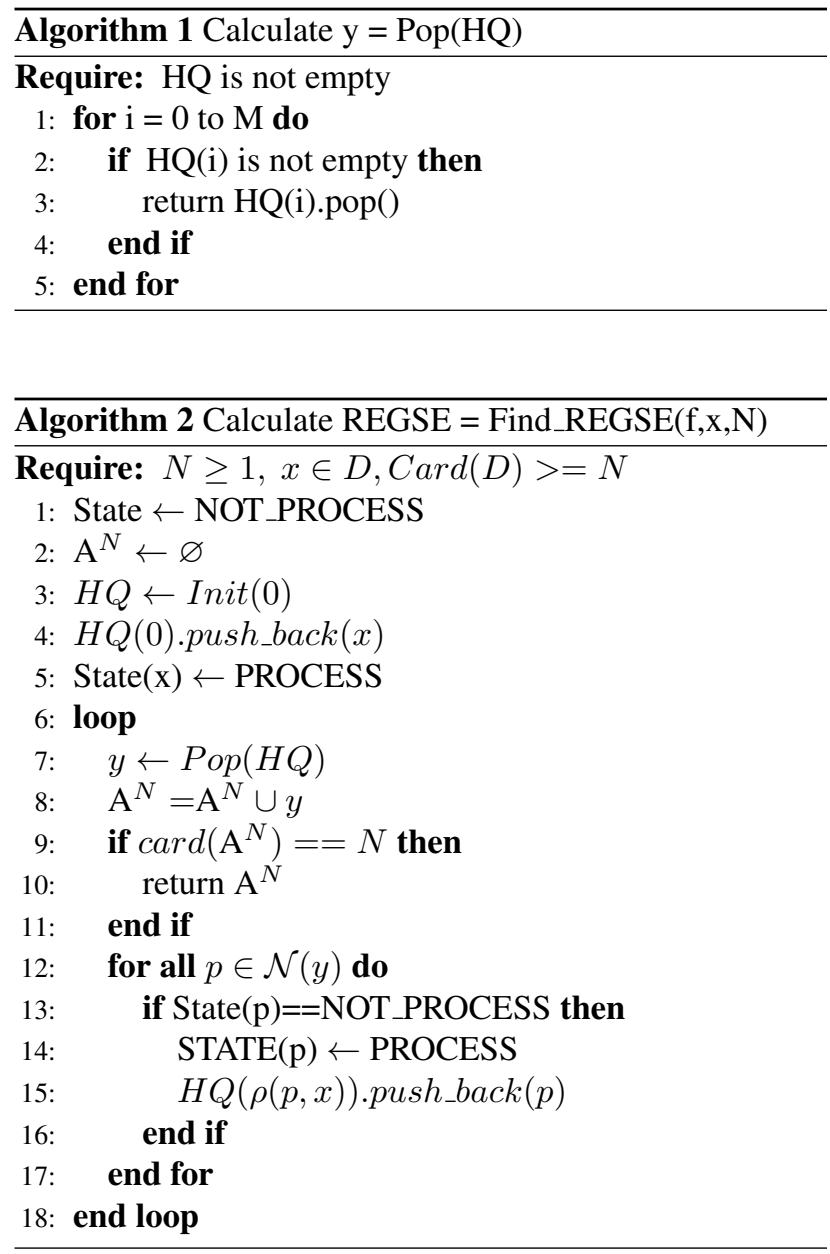

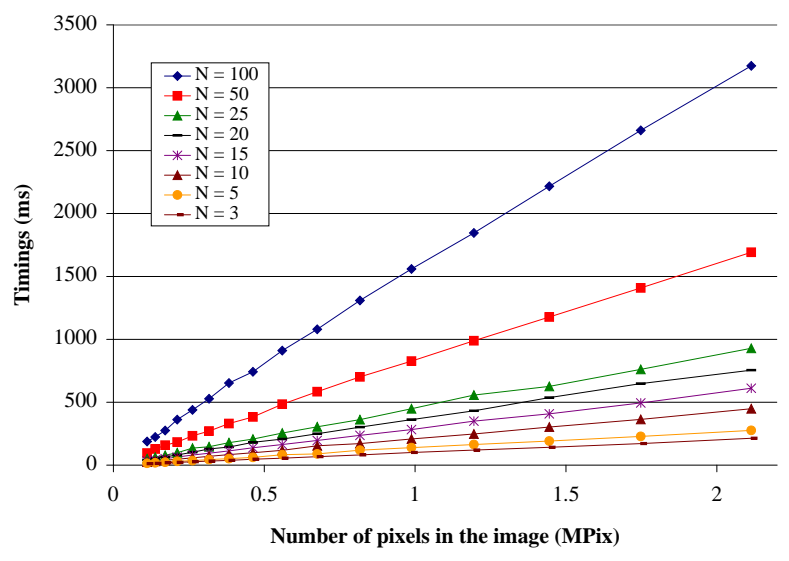

Figure 8. Linear w.r.t. $n$ : Computation time of an erosion with REGSEs as function of the number of pixels in the image and for different size of SEs. This benchmark was made on a computer Intel Core (i7-870) CPU @ $2.93 \mathrm{GHz}$ using a multithreaded $\mathrm{C}++$ implementation. (Images: Lena of increasing size)

\subsection{Implementation}

The computation of the REGSE's shape is described in algorithm 2. This function returns a set of $N$ pixels corresponding to $\mathrm{A}^{N}$. We consider the $N$ first pixels that are popped from the HQ as our structuring element (Line 8). Using a HQ avoids a computationally intensive sorting step and figure 8 shows that the computation time is linear with reference to the number of pixels of the image and with the size of the structuring element.

\subsection{Comparison with amoebas and GANSE}

In comparison with amoebas, the complexity of REGSEs is almost the same. In practice however, the construction of REGSEs is faster than amoebas. This is due to the computation of the amoebas' distance, which is time consuming in comparison with the extraction of the closest pixels in gray-scale for the REGSEs.

The complexity of GANSE's algorithm is more difficult to handle as it depends on the value of the parameter $m$ and on the content of the image. There is no size constraint and each structuring element can potentially grow up through the entire image. In the general case however, GANSEs are usually much slower than REGSEs.

We store the computation time required to build figure 6. We use only one core of our Intel Core 2 Duo (T7700) CPU, @2.4GHz and we use the same image processing library. We get $0.87 s, 2.05 s$ and $201 s$ respectively for computing an opening with REGSEs, amoebas and GANSEs. 


\section{Conclusion and future work}

We have presented here new adaptive structuring elements used to build morphological operators. The structuring elements can have any shape as long as it is a connected component, with a fixed area. This is the main advantage of filters based on REGSE. Any one-pixel wide structure can be extracted, whereas other structuring elements cannot fit in the object and therefore destroy it. All classical morphological operators based on structuring elements can be used with REGSEs. Openings and closings, with region growing structuring elements offer possibilities of very fine filtering, while preserving the boundaries of the image. The parameterization of this algorithm is also very easy. The only parameter to handle is the number of pixel of the structuring elements. This is a clear advantage compared with other filters.

Furthermore, we introduce new and efficient tools to enhance thin structures of the image. To the authors' knowledge, this is the first time that the shape of the SEs is analyzed to obtain some features. The Inertia and Extension operators are powerful tools and they offer many new possibilities. These operators are built for REGSEs but they can be adapted to all other adaptive structuring elements.

These operators are faster than GANSE and amoebas and they are fast enough for many applications. However, we are currently working on a fast and accurate approximation of the REGSEs and on another representation of the image, which is based on graphs, to speed up the computation time.

Future work will include the extension of these filters to color images. Classically, we could apply independently, a filter on each channel and then combine the results to obtain the filtered image. The use of more perceptual distances in the LAB or HLS color space, would improve the quality of the result by reducing the false color artifacts. We will also extend the operators based on the shape of the SEs.

This framework is general as we can apply it to linear and non linear filters with images of any dimension $(1 \mathrm{D}, 2 \mathrm{D}, \ldots, \mathrm{nD})$. Therefore, it offers new perspectives for noise reduction and features enhancement.

\section{Acknowledgments}

This work was made possible thanks to the support of the "Pôle ASTech" and the "Pôle Nucléaire de Bourgogne", and has been financed by the French "département de Seine et Marne"

The authors are grateful to Ms Raviart, working in the "Centre des matériaux, MINES ParisTech", for help with the optical microscope.

\section{References}

[1] S. Beucher, J. Blosseville, and F. Lenoir. Traffic spatial measurements using video image processing. Intelligent Robots and Computer Vision, Proc. SPIE, 848:648-655, 1987.

[2] N. Bouaynaya, M. Charif-Chefchaouni, and D. Schonfeld. Theoretical foundations of spatiallyvariant mathematical morphology part I: Binary images. IEEE transactions on pattern analysis and machine intelligence, 30(5):823, 2008.

[3] N. Bouaynaya and D. Schonfeld. Theoretical foundations of Spatially-Variant mathematical morphology part II: Gray-Level images. IEEE Trans. Pattern Anal. Mach. Intell., 30(5):837-850, 2008.

[4] U. Braga-Neto. Alternating sequential filters by Adaptive-Neighborhood structuring functions. In International Symposium on Mathematical Morphology and Its Applications to Image and Signal Processing, pages 139-146, Atlanta, May 1996. P. Maragos, R.W. Schafer and M.A. Butt.

[5] F. Cheng and A. Venetsanopoulos. An adaptive morphological filter for image processing. IEEE Transactions on Image Processing, 1(4):533-539, 1992.

[6] J. Debayle and J. Pinoli. General adaptive neighborhood image processing. Journal of Mathematical Imaging and Vision, 25(2):245-266, 2006.

[7] R. Gordon and R. Rangayyan. Feature enhancement of film mammograms using fixed and adaptive neighborhoods. Applied Optics, 23(4):560-564, 1984.

[8] J. Grazzini and P. Soille. Adaptive morphological filtering using similarities based on geodesic time. In Discrete Geometry for Computer Imagery, pages 519-528. Springer, 2008.

[9] R. Lerallut, É. Decencière, and F. Meyer. Image filtering using morphological amoebas. Mathematical Morphology: 40 Years On, pages 13-22.

[10] P. Salembier. Structuring element adaptation for morphological filters. Journal of visual communication and image representation, 3(2):115-136, 1992.

[11] J. Serra. Image analysis and mathematical morphology. Academic, London, 1, 1982.

[12] J. Serra. Image analysis and mathematical morphology. Academic, London, 2 Theoretical Advances, 1988.

[13] O. Tankyevych, H. Talbot, P. Dokladal, and N. Passat. Spatially-Variant Morpho-Hessian Filter: Efficient Implementation and Application. In Mathematical Morphology and Its Application to Signal and Image Processing, page 137. Springer-Verlag New York Inc, 2009. 